\section{Manuel Mark \\ Thomas Lufkin \\ Andrée Dierich \\ Marianne LeMeur \\ Pierre Chambon}

\title{
Inactivation du gène Hox-1.6 chez la souris : vers le décodage des réseaux d'homéogènes de mammifères
}

La preuve directe de la participation des gènes Hox à l'ontogenèse a été acquise au cours des derniers mois grâce à la production d'animaux transgéniques porteurs d'homéogènes modifiés. La stratégie retenue dans le cas de Hox-1.6 a consisté à l'inactiver par recombinaison homologue dans les cellules souches embryonnaires de souris. Elle a permis l'obtention d'homozygotes létaux dont les anomalies, strictement limitées à la partie postérieure du cerveau et du crâne, ont été analysées. Contrairement à ce qui est observé chez la drosophile, les malformations ne ressemblent pas à des transformations homéotiques.

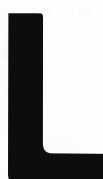

'organisme des êtres pluricellulaires est un système coopératif constitué d'entités fonctionnelles et structurales : les tissus et les organes. Comprendre les mécanismes moléculaires contrôlant dans l'espace et dans le temps la morphogenèse et les cytodifférenciations constitue le problème clé de la biologie du développement. Comme tout organisme provient d'une seule cellule primordiale, le plus souvent l'œuf fécondé, ses cellules sont, sauf exceptions, génétiquement identiques. Il faut donc admettre, en schématisant beaucoup, que l'édification d'un organisme requiert l'activation et/ou la répression séquentielles et coordonnées de gènes.

* Gène homéotique : gène impliqué dans des mutations homéotiques, remplacant un segment du corps par un autre.

** Homéogène : gène contenant une région codant pour un domaine appelé "homéo ", région polypeptidique d'une soixantaine d'acides aminés adoptant une configuration de type "hélice-tour-hélice" et se liant à des séquences spécifiques d'ADN. Les gènes homéotiques sont des homéogènes; tous les homéogènes ne sont pas, nécessairement, des gènes homéotiques. Les homéogènes mentionnés dans cet article appartiennent tous
L'analyse génétique de certaines mutations affectant le développement chez la drosophile (mouche du vinaigre) a permis la découverte d'un ensemble de gènes qui gouvernent la mise en place du plan d'organisation corporel de l'insecte [1]. Parmi eux, les gènes homéotiques* (gènes $H O M$, chez la drosophile) dont les produits (ou homéoprotéines), distribués le long de l'axe antéro-postérieur (rostro-caudal) de l'embryon, jouent un rôle majeur dans la détermination de l'identité spatio-temporelle des cellules. Les gènes $H O M$ sont organisés en un complexe, appelé HOM-C, porté par le chromosome 3 (figure 1). Grâce à des sondes nucléotidiques dérivées des gènes $H O M$, des homéogènes** ont été identifiés chez de nombreuses espèces animales, incluant les mammiferes [2]. Les homéogènes de mammiferes, appelés Hox, sont, pour la plupart, groupés en quatre complexes homologues, $H O X-1,-2,-3$ et -4 , chacun localisé sur un chromosome différent [3]. Les complexes d'homéogènes de mammifères présentent la même organisation que le complexe prototype HOM-C : à chaque gène $H O M$ correspondent jusqu'à quatre gènes $H o x$, appelés gènes paralogues, qui présentent entre eux de très fortes similitudes de 


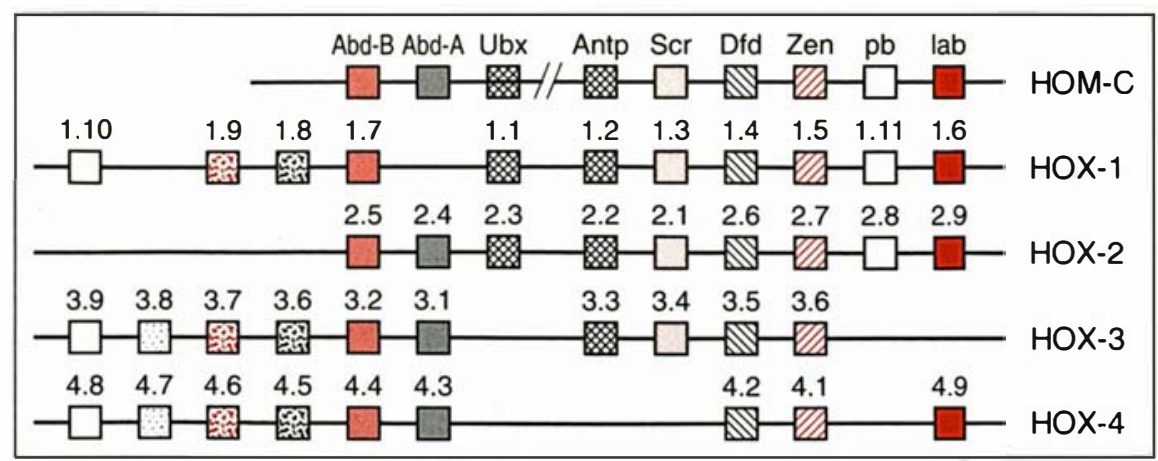

Figure 1. Représentation schématique des complexes d'homéogènes de la drosophile (HOM) et des mammifères (HOX). La comparaison des séquences nucléotidiques permet d'aligner verticalement les gènes semblables (paralogues). Abd-A et Abd-B, abdominal $A$ et $B$; Ubx, ultrabithorax ; Antp, antennapedia ; Scr, sex combs reduced; Dfd, deformed; Zen, zerknüllt ; pb, proboscipedia ; lab, labial. Les gènes Hox sont identifiés par le numéro du complexe suivi d'un chiffre qui tient compte de l'ordre dans lequel ces gènes ont été isolés.

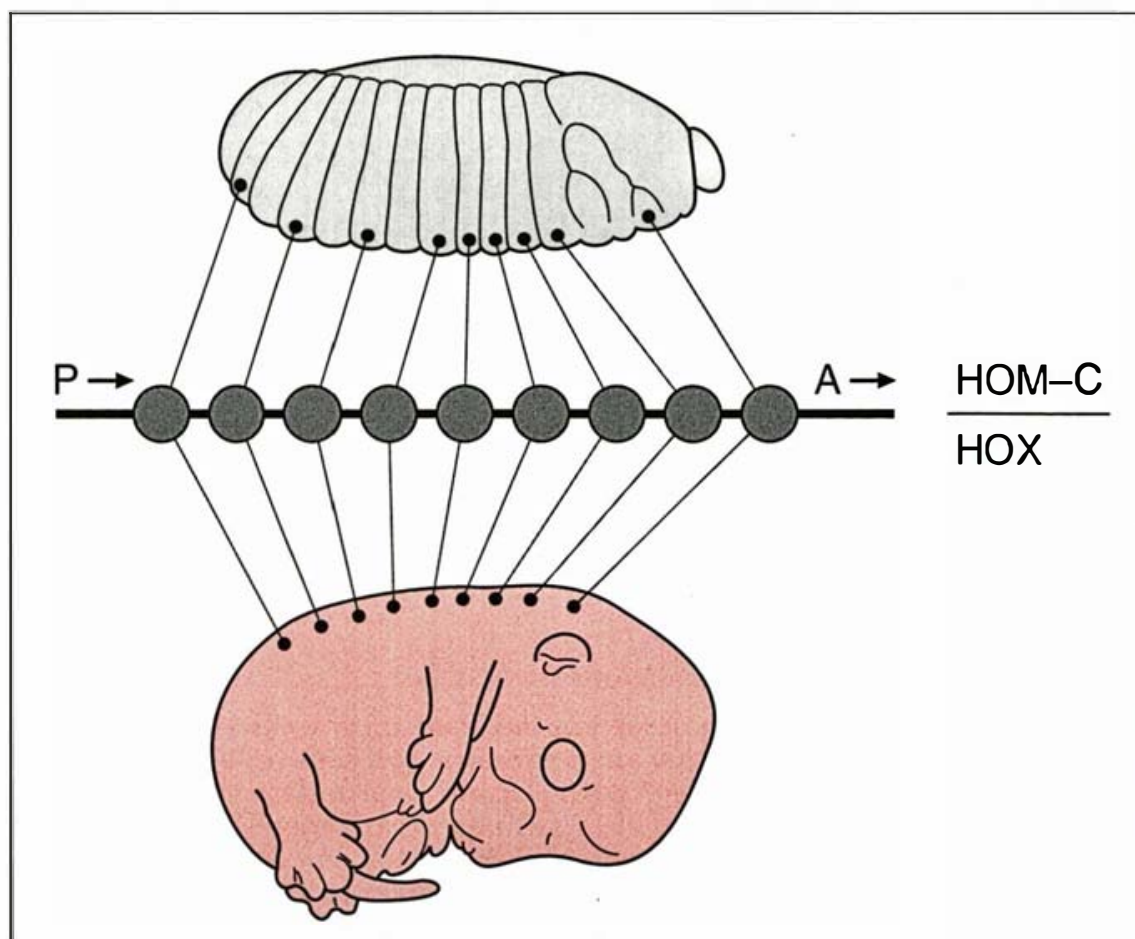

Figure 2. Règle de colinéarité. En partant du début d'un complexe, représenté conventionnellement à droite sur le chromosome, et en se dirigeant vers la gauche, on trouve des homéogènes dont l'expression le long de l'axe antéropostérieur de l'embryon (mouche en haut, souris en bas) est restreinte à un territoire corporel de plus en plus postérieur (mouche) ou dont la frontière d'expression antérieure est de plus en plus décalée vers la région caudale (souris). Cette règle souffre certaines exceptions (voir figure 5).

$\mathrm{m} / \mathrm{s} n^{\circ} 4$, vol. 8 , avril 92 séquences et occupent les mêmes positions relatives à l'intérieur de chacun des quatre complexes (figure 1). De plus, l'expression spatiale des homéogènes d'un complexe donné obéit aux mêmes règles chez la drosophile et chez la souris : il existe une relation linéaire entre la position de ces gènes sur le chromosome et la frontière antérieure de leurs territoires d'expression le long de l'axe antéro-postérieur de l'embryon (figure 2) [4]. La conservation au cours de l'évolution, à la fois, de l'organisation des homéogènes et de leur expression spatiale a conduit à l'hypothèse qu'ils pourraient remplir des fonctions analogues chez des espèces dont les stratégies du développement sont, en apparence, totalement différentes. Chez la souris, les mutations homéotiques sont rares. $\mathrm{La}$ preuve directe de l'implication des gènes Hox dans un processus de développement passe donc par la production d'animaux transgéniques. Dans la pratique, cela revient soit à muter, par recombinaison homologue, un homéogène donné, soit à intégrer, au hasard, dans le génome, une copie supplémentaire du gène pouvant s'exprimer dans un territoire ectopique de l'embryon [5-7].

La première approche a été appliquée récemment au gène $H o x-1.5 \mathrm{~m} / \mathrm{s}$ $n^{\circ}$ 6, vol. 7, p. 618) [6] et, dans notre propre laboratoire, au gène $H o x-1.6$ [7]. Le gène $H o x-1.6$, isolé à partir d'une banque génomique de souris, a d'abord été inactivé par délétion d'une partie de sa séquence nucléotidique, puis il a été introduit, par recombinaison homologue, dans le génome de cellules souches embryonnaires (ES) de souris pour se substituer au gène normal. Ces cellules ES contenant un allèle Hox-1.6 muté ont été ensuite utilisées pour " construire " des souris homozygotes (Hox-1.6-1- $)$ ne produisant plus l'homéoprotéine correspondante.

\section{L'inactivation des deux allèles du gène Hox-1.6 est létale à la nais- sance}

Une portée de souriceaux issus du croisement de parents hétérozygotes (Hox-1.6+1-) comporte, en moyenne, un individu sur quatre qui, incapable de respirer, meurt d'anoxie dans les minutes qui suivent la naissance. 


\section{RÉFÉRENCES}

1. Gehring WJ. Homeoboxes in the study of devclopment. Science 1987 ; 236 : 1245-52.

2. Akam M. Hox and HOM : homologous gene clusters in insects and vertebrates. Cell $1989 ; 57$ : 347-9.

3. Duboulc D, Dollé P, Gaunt SJ. Les gènes du développement des mammifères. La Recherche 1990; 21 : 294-303.

4. Jacob F. L'unité du vivant. médecine/sciences $1990 ; 6: 222-7$.

5. Balling R, Mutter G, Gruss P, Kessel M. Cranofacial abnormalitics induced by ectopic expression of the homeobox genc Hox-1.1 in transgenic mice. Cell $1989 ; 58$ 337-47.

6. Chisaka O, Capecchi MR. Regionally restricted developmental defects resulting from targeted disruption of the mouse homeobox gene Hox-1.5. Nature 1991; 350 : 473-9.

7. Lufkin T, Dicrich A, LcMcur M, Mark M, Chambon P. Disruption of the Hox-1.6 homeobox gene results in defects in a region corresponding to its rostral domain of expression. Cell $1990 ; 66: 1-20$.

8. Lumsden A. The cellular basis of segmentation in the developping hindbrain. Trends Neurosci $1990 ; 13: 329-35$.

9. Murphy P, Hill RE. Expression of the mouse labial-like homeobox-containing genes, Hox-2.9 and Hox 1.6, during segmentation in the hindbrain. Development $1991 ; 111: 61-74$

10. LeDouarin NM, Teillet MA, Couly G Chimères embryonnaires et développement du système nerveux. médecine/sciences 1990 $6: 228-44$.

11. Noden DM. Interactions and fates of avian craniofacial mesenchyme. Development 1988 ; 103 (suppl.) : 121-40.

12. Chisaka O, Musci TS, Capccchi MR Developmental defects of the ear, cranial nerves and hindbrain resulting from targeted disruption of the mouse homeobox gene Hox-1.6. Nature 1992 ; 355 : 516-20.

13. Hunt P, Krumlauf R. Deciphering the Hox code : clues to patterning branchial
Ces nouveau-nés ne se différencient pas des autres individus de la portée par leur morphologie externe, mais l'analyse de leur ADN, par la technique de Southern, révèle qu'ils sont porteurs du gène recombiné à l'état homozygote [7].

L'examen des squelettes et de coupes histologiques sériées des fœtus Hox-1.6-1- révèle des ano-

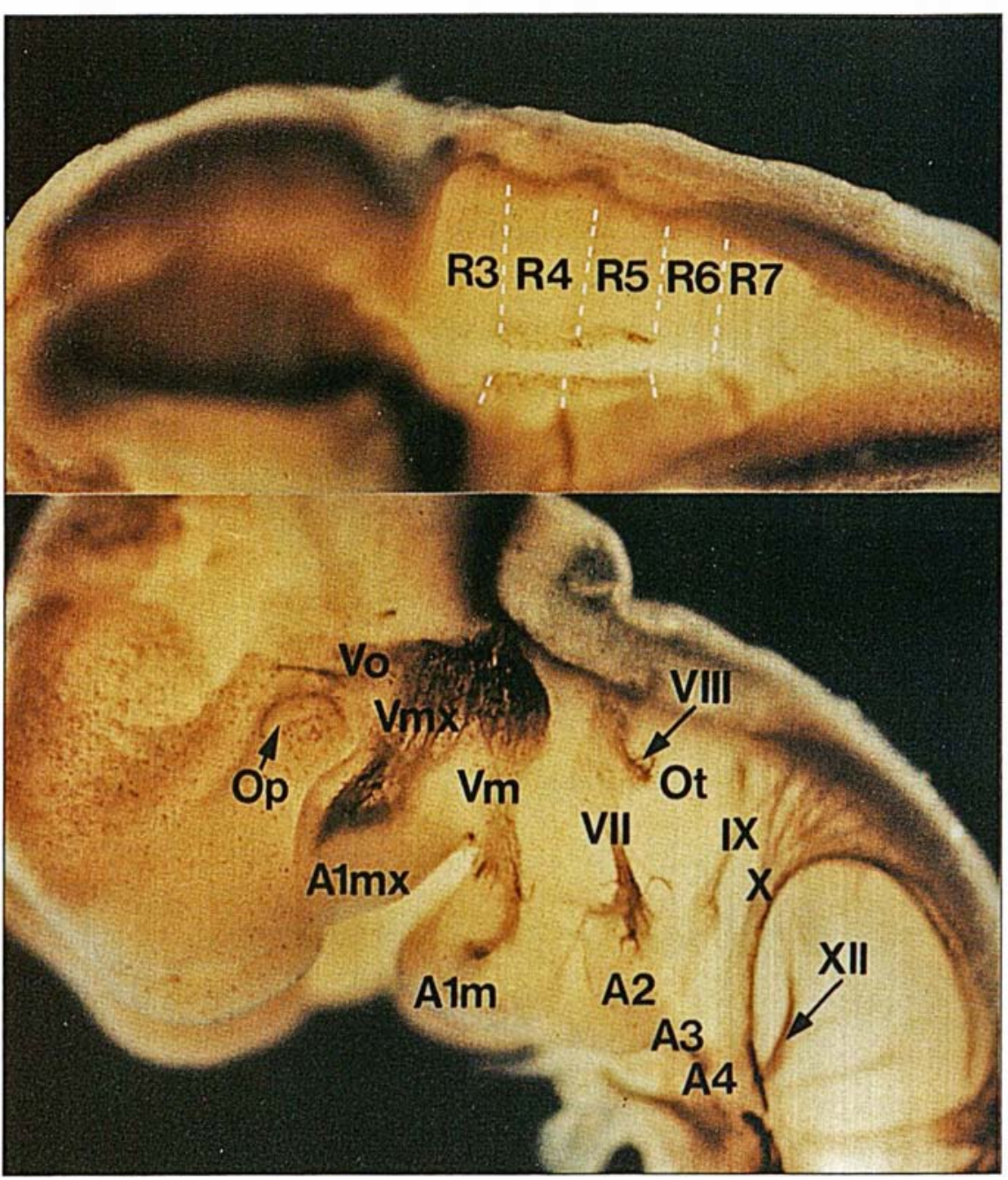

Figure 3. Vue dorsale (en haut) et vue latérale d'un embryon normal de souris au jour 10,5, marqué par un anticorps anti-neurofilaments. Le toit du cerveau postérieur a été enlevé partiellement pour montrer certains rhom bomères (R3-7). Le nerf trijumeau (V), issu des rhombomères 2 et 3, innerve les portions maxillaire $(A 1 \mathrm{mx})$ et mandibulaire $(A 1 \mathrm{~m})$ du 1 er arc pharyngien. Le nerf facial (VII), issu des rhombomères 4 et 5 , innerve le 2e arc pharyngien (A2). Les nerfs glosso-pharyngien $(I X)$ et vague $(X)$, issus des rhombomères 6 à 8 , sont, respectivement, les nerfs des $3^{e}$ et $4^{e}$ arcs pharyngiens (A3 et A4). Les nerfs stato-acoustique (VIII) et grand hypoglosse (XII) sont dérivés respectivement des rhombomères 4 et 8 . Le mésenchyme des arcs pharyngiens (en jaune sur la photo) est lui-même un dérivé des crêtes neurales du cerveau postérieur (A1m, A2, A3, A4) ou du cerveau moyen ( $11 \mathrm{mx}$ ). Vo, $V m x, V m$, respectivement : rameaux ophtalmique, maxillaire et mandibulaire du V; Op, ébauche de l'œil ; Ot, ébauche de l'oreille interne. (Don du

$\operatorname{Dr} A$. Lumsden.) malies multiples, mais intéressant Des fœtus Hox-1.6 $6^{-1-}$ âgés de 15 et 18,5 jours ont été examinés (chez la Chez ces foetus, la partie basale de l'os occipital (basi-occipital) est déformée. La morphologie de l'oreille interne (organe de l'équilibration et de l'audition) est considérablement 
altérée ainsi que celle du rocher, partic de l'os temporal entourant l'oreille interne. Le noyau moteur du nerf facial (VIIe paire de nerfs crâniens, VII) est absent. La taille des ganglions sensitifs des nerfs statoacoustique (VIII), glosso-pharyngien (IX) et vague $(\mathrm{X})$ est très réduite. Enfin, les filets nerveux qui - chez l'embryon normal - connectent ces ganglions au cerveau sont absents [7]. C'est également au niveau du cerveau postérieur que se situent les centres respiratoires dont les influx, relayés en partie par le nerf vague, assurent la commande coordonnée des mouvements respiratoires. Leurs neurones, qui ne forment pas d'agrégats anatomiquement définis, ne peuvent être identifiés sur une coupe histologique. Néanmoins, la lésion de ces centres respiratoires associée à celle des nerfs vagucs apparaît comme la cause la plus probable de la mort des nouveau-nés homozygotes.

Les structures affectées par la mutation dérivent d'un territoire corporel correspondant aux rhombomères $4,5,6$ et 7

Chez l'embryon de souris âgé de 8,5 à 11 jours, les rhombomères correspondent à des dilatations du cerveau postérieur séparées par des sillons, particulièrement bien visibles et initialement décrits chez le poulet. Ils reflètent une organisation transitoire du cerveau postérieur en segments. Cette segmentation impose les positions relatives des noyaux moteurs et des ganglions sensitifs des nerfs crâniens ainsi que celle des arcs pharyngiens chez le fotus et chez l'animal adulte (figures 3 et 4) [8]. Les noyaux moteurs des nerfs crâniens se différencient in situ, dans l'épaisseur même du neurectoderme (tissu nerveux de l'embryon). Au niveau des rhombomères 4-7, il s'agit des noyaux moteurs des nerfs VII (dérivés des rhombomères 4 et 5 ), VI (rhombomères 5 et 6 ) et IX (rhombomères 6 et 7). Le premier, de grande taille, peut être facilement identifié sur une coupe histologique chez de jeunes fotus normaux ou hétérozygotes Hox-1.6 $6^{+1-}$

Lcs ganglions sensitifs des nerfs crâniens et le mésenchyme des arcs pharyngiens proviennent, pour $\mathrm{m} / \mathrm{s} n^{\circ} 4$, vol. 8 , avril 92 l'essentiel, des crêtes neurales céphaliques. Les cellules des crêtes neurales céphaliques s'individualisent dans le neurectoderme avant de migrer, parfois sur de longues distances. Leurs potentialités sont multiples [10, 11]. Les cellules des crêtes neurales originaires du cerveau postérieur migrent en deux vagues successives. Dans la région des rhombomères 4-7 (figure 4), la vague migratrice du jour

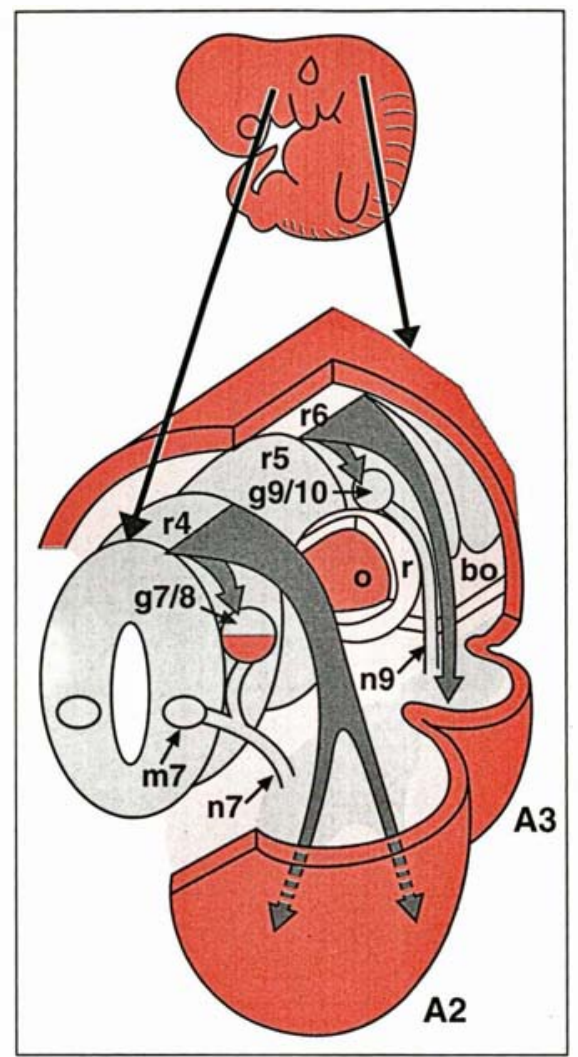

Figure 4. Schéma montrant l'origine embryologique normale des différentes structures dans la région affectée par l'inactivation du gène Hox-1.6. En rouge, l'ectoderme et ses dérivés : oreille interne épithéliale (o) et une partie du complexe ganglionnaire VII-VIII (g7/8). En gris, les structures dérivées du neurectoderme : parois des rhombomères 4, 5 et $6(r 4, r 5$ et $r 6)$ où se différencie le noyau moteur du nerf VII ( $m 7)$; cellules des crêtes neurales qui, après migration (flèches noires), participent à l'édification des complexes ganglionnaires VII-VIII et IX-X (g9-10) et à la formation des $2^{e}$ et $3^{e}$ arcs pharyngiens (A2 et A3). En rose, le mésoderme dont dérivent l'os basi-occipital (bo) et le rocher (r). $n 7$ et $n 9$, respectivement: nerfs facial et glossopharyngien. (D'après [13])
8 édifie les $2^{e}, 3^{e}$ et $4^{e}$ arcs pharyngiens, celle du jour 9 donne naissance aux ganglions sensitifs des VIIe VIIIe, IX $^{\mathrm{e}}$ et $\mathrm{X}^{\mathrm{e}}$ paires de nerfs crâniens. Seules les cellules des crêtes neurales destinées à ces ganglions de nerfs crâniens paraissent affectées par l'inactivation de Hox-1.6 ; les structures dérivées de la première vague de migration ne semblent pas altérées chez les sujets Hox-1.6-1- Enfin, la taille et la position relative le long de l'axe rostro-caudal des structures nerveuses issues des rhombomères 2 et 3 (noyau moteur et ganglion sensitif du nerf trijumeau, V), et 8 (noyau moteur du nerf grand hypoglosse, XII) sont normales chez les fœtus Hox-1. $6^{-1-}$

Les anomalies des fœtus Hox$1.6^{-1-}$ qui sont limitées au territoire antérieur d'expression initiale du gène, sont déterminées précocement et peuvent s'expliquer par un défaut de spécification des cellules du neurectoderme

Chez l'embryon normal [9], le territoire d'expression de $H o x-1.6$ visualisé par hybridation in situ s'étend, au jour 8 de la gestation, depuis la limite antérieure du futur rhombomère 4 jusqu'à l'extrémité caudale de l'embryon. En l'espace de quelques heures, l'expression du gène dans le cerveau postérieur cessera de façon définitive (figure 5, p. 338). Cependant, le gène restera exprimé dans les régions plus postérieures du corps de l'embryon jusqu'à la fin de la période embryonnaire. Aucune anomalie n'est observée dans les tissus et organes dérivés de ces territoires d'expression tardive du gène.

$\mathrm{Au}$ jour 8 , le neurectoderme contient les précurseurs des neurones moteurs du nerf facial, ainsi que ceux des cellules des crêtes neurales à l'origine des ganglions sensitifs des nerfs crâniens. Par ailleurs, il induit la formation, à partir de l'ectoderme, de l'oreille interne épithéliale (qui n'exprime pas Hox-1.6). Plus tard, celle-ci "instruit " la formation du rocher à partir de mésoderme (tissu conjonctif indifférencié de l'embryon). Enfin, l'os basi-occipital se développe au contact du rocher et sa croissance est en partie régie par des contraintes mécaniques. Il est bon de souligner toutefois qu'il s'agit là d'une 


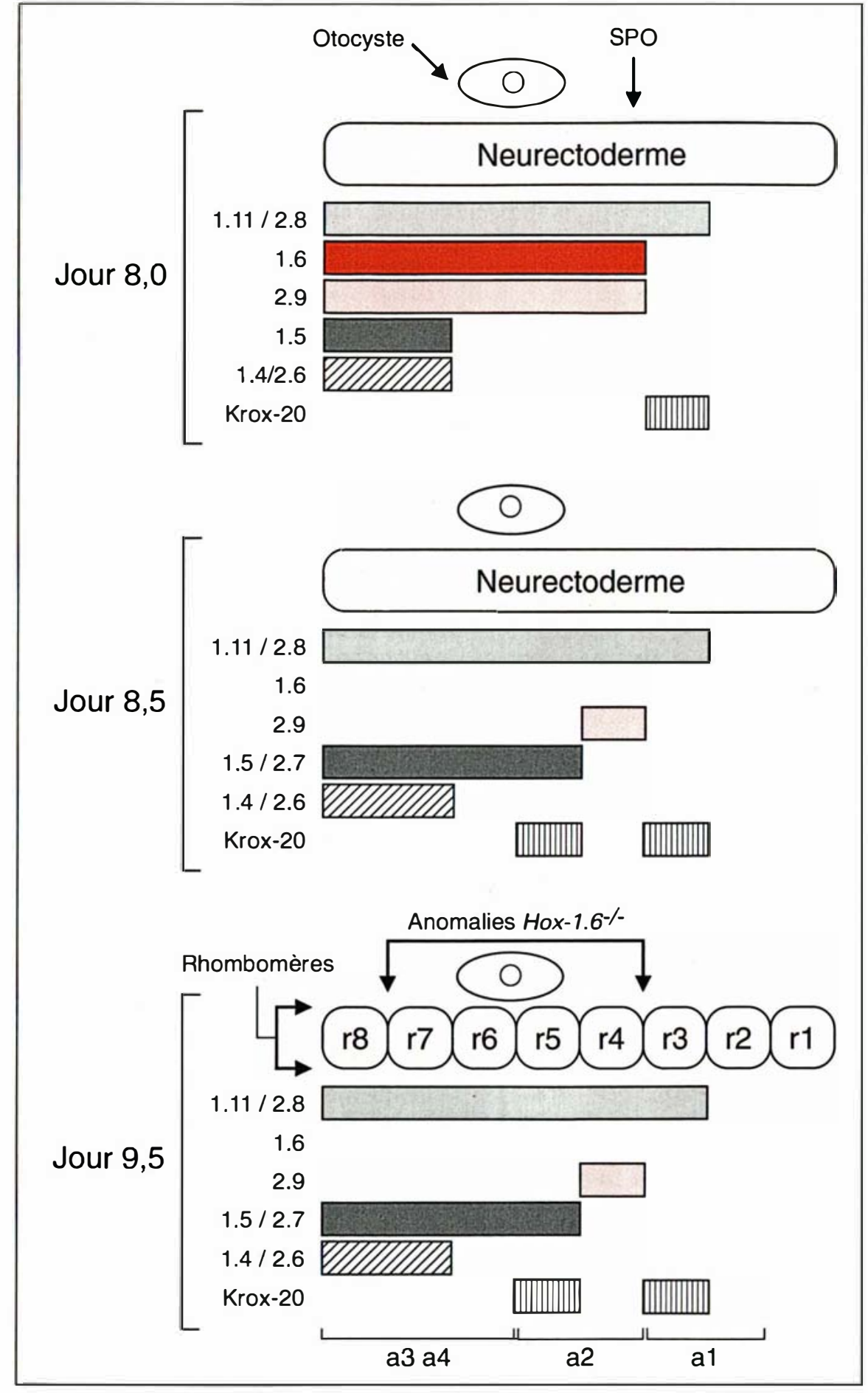

Figure 5. Représentation schématique de l'expression de certains des gènes des complexes $H O X-1$ et $H O X-2$ dans le cerveau postérieur à différents stades du développement chez l'embryon de souris déterminée par la technique d'hybridation in situ. Noter que la série complète des rhombomères ( $r 1$ à $r 8$ ) n'est établie qu'au jour 9. La détermination des frontières antérieures d'expression aux jours 8 et 8,5 utilise des repères morphologiques (par exemple le sulcus pré-otique : SPO) et moléculaires (l'expression du gène Krox 20I ; la plupart des autres gènes Hox (voir figure 1), ne sont pas exprimés dans le cerveau postérieur. 0, ébauche de l'oreille interne ; a 1-a4, arcs hypothèse embryologique et de rappeler que les cellules du mésoderme céphalique expriment Hox-1.6 au même titre que celles du neurectoderme. On notera également que la conséquence morphologique la plus précoce de l'inactivation de Hox-1.6 est un retard de la fermeture du tube neural au niveau du cerveau postérieur des embryons homozygotes. Cette manifestation, à dominance inconstante, est observée au jour 9,5 (figure 6). Dans l'état actuel des connaissances embryologiques, il est impossible d'établir un lien causal entre cette fermeture différée et les anomalies observées au stade fœtal.

L'étude des mutants Hox " nuls " fournit de précieux renseignements sur le rôle des homéogènes mammifères et sur la logique fonctionnelle du code Hox

Le phénotype des fotus Hox-1.6-1[7] diffère complètement de celui des foetus Hox-1.5-1- [6]. La frontière antérieure d'expression de Hox-1.5, établie au jour 8,5 , correspond à la limite antérieure du futur rhombomère 5 (figure 5). Les homozygotes Hox-1.5-1- dont les nerfs crâniens et l'oreille interne sont normaux, montrent des déficits multiples parmi les structures dérivées des arcs pharyngiens. Il semblerait donc que deux gènes Hox différents appartenant au même complexe et exprimés successivement dans une même région du cerveau postérieur puissent déterminer le devenir des cellules de la crête neurale, avant leur migration, vers une destinée soit neurale (ganglions nerveux), soit mésenchymateuse (arcs pharyngiens).

Le complexe prototype HOM-C a été quadrupliqué chez les vertébrés, et la forte conservation, au cours de l'évolution, des séquences nucléotidiques des gènes paralogues a fait évoquer la possibilité de redondances fonctionnelles. A l'opposé, il a été maintes fois suggéré que la construction d'une région corporelle spécifique au cours de l'ontogenèse requerrait l'intégration des actions de toutes les homéoprotéines exprimées dans cette région. Ce modèle combinatoire de code Hox implique, dans sa formulation extrême, que l'inactivation d'un seul gène Hox altère le développement de l'ensemble des tissus et organes du 


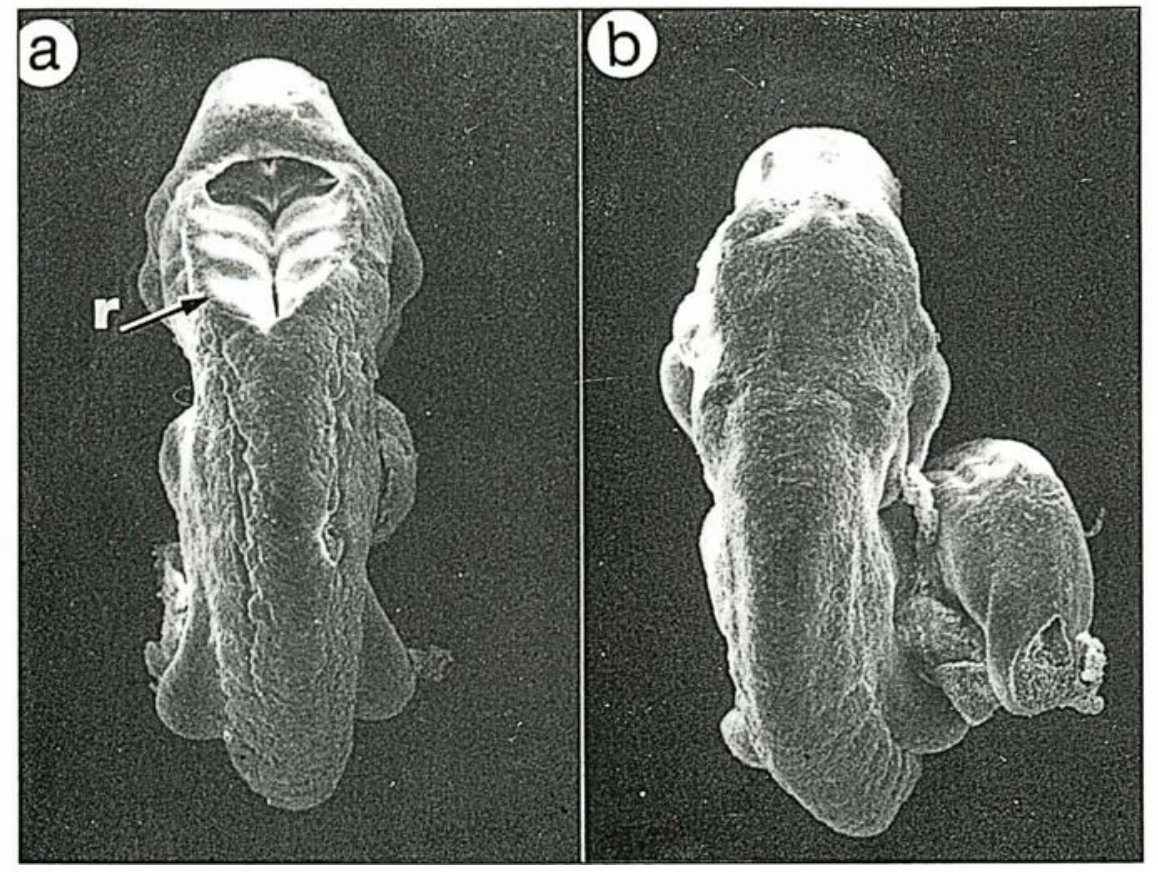

Figure 6. Vue dorsale d'embryons de souris âgés de 9,5 jours, examinés au microscope électronique à balayage. (a) un sujet homozygote, Hox$1.6^{-1-}$ : I'ouverture persistante du tube neural permet de voir les rhombomères (r). (b) un sujet hétérozygote Hox-1.6 $6^{+1-}$.

territoire d'expression du gène. Nos observations démontrent clairement que l'inactivation de $H o x-1.6$ affecte uniquement certains organes dérivés de son territoire antérieur d'expression initiale.

La production d'anomalies phénotypiques en réponse à l'inactivation du seul gène $H o x-1.6$, confirmées pour l'essentiel dans un récent article du laboratoire de M.R. Capecchi [12] signifie que certaines de ses fonctions ne peuvent être assurées par ses paralogues des complexes HOX-2 et HOX-4 et, de façon plus générale, que les gènes $H o x$ coexprimés au jour 8 dans le territoire antérieur d'expression initiale de $H o x-1.6$, c'està-dire $H o x-2.8, H o x-2.9$ et $H o x-1.11$ (voir figure 5), ne sont pas, du moins complètement, redondants. Des conclusions similaires ont été tirées de l'observation des fotus Hox-1.5 -1[6]. Le modèle combinatoire de code Hox s'avère donc partiellement valide dans les régions antérieures d'expression de Hox-1.6 et de Hox-1.5. En revanche, les homéoprotéines codées par Hox-1.6 et par Hox-1.5 ne paraissent pas jouer un rôle dans la morphogenèse des régions de l'embryon $m / s n^{\circ} 4$, vol. 8, avril 92

\section{Summary}

Disruption of the Hox-1.6 hemeobox gene results in defects in a region corresponding to its rostral domain of expression

Direct proof for the partıcipation of mouse homeobox-containing genes in embryonic development has been shown recently by the generation, via targeted gene disruption, of mutant mice which lack a functional homeoboxcontaining gene. In the case of the homeobox-containing gene Hox1.6, mice heterozygous (Hox$\left.1.6^{+1-}\right)$ for the mutated Hox-1.6 gene are normal, however homozygous Hox-1.6-1- mice die immediately after birth from anoxia. Analysis of Hox-1.6-1mice at various stages of development show numerous defects which include : delayed closure of the neural tube ; absence of certain cranial nerve roots, and nuclei ; altered cranial ganglia ; malformed inner ears; and malformed bones of the skull. Although Hox-1.6 has a large expression domain in early embryos, defects are located only in its most rostral region of expression. In contrast to what has been observed in Drosophila, the defects in Hox-1.6 $6^{-1}$ homozygotes do not appear to resemble a homeotic transformation. The mechanism for the mode of action of mouse homeobox-containing genes is discussed. ment d'un autre segment. Il est clair que les manifestations phénotypiques de l'inactivation de $H o x-1.6$ ne correspondent pas à une telle transformation, qui pourrait dans ce cas nécessiter l'inactivation simultanée des gènes paralogues.

Il existe chez la souris une trentaine d'homéogènes, tous candidats potentiels à une inactivation par recombinaison homologue dans des cellules souches embryonnaires. Dans ce contexte, il convient de souligner que l'analyse phénotypique des animaux transgéniques n'est qu'un élément d'un programme de recherche dont les objectifs sont d'identifier les gènes cibles des homéoprotéines et d'élucider la manière dont leur expression est contrôlée
Remerciements

Les auteurs remercient le professeur JeanVictor Ruch pour sa lecture critique du manuscrit, ainsi que Mme Christiane Werlé pour son aide à la réalisation des illustrations.

TIRÉS A PART 